\title{
A!
}

This is an electronic reprint of the original article.

This reprint may differ from the original in pagination and typographic detail.

Moilanen, Antti; Hakala, Tommi; Törmä, Päivi

\section{Active Control of Surface Plasmon-Emitter Strong Coupling}

\section{Published in:}

ACS Photonics

DOI:

10.1021/acsphotonics.7b00655

Published: 12/12/2017

Document Version

Peer reviewed version

Please cite the original version:

Moilanen, A., Hakala, T., \& Törmä, P. (2017). Active Control of Surface Plasmon-Emitter Strong Coupling. ACS

Photonics, 5(1), 54-64. https://doi.org/10.1021/acsphotonics.7b00655

This material is protected by copyright and other intellectual property rights, and duplication or sale of all or part of any of the repository collections is not permitted, except that material may be duplicated by you for your research use or educational purposes in electronic or print form. You must obtain permission for any other use. Electronic or print copies may not be offered, whether for sale or otherwise to anyone who is not an authorised user. 


\title{
Active Control of Surface Plasmon - Emitter Strong Coupling
}

\author{
Antti J. Moilanen, Tommi K. Hakala, and Päivi Törmä* \\ COMP Centre of Excellence, Department of Applied Physics, Aalto University School of \\ Science, FI-00076 Aalto, Finland \\ E-mail: paivi.torma@aalto.fi
}

\begin{abstract}
Strong coupling between quantum emitters and surface plasmon polariton modes in metal nanostructures has been extensively studied in recent years. A natural direction of research and a prerequisite for many applications is the possibility of external, in-situ manipulation of the strength of the coupling. We review research on active control of surface plasmon - emitter strong coupling phenomena. Active control has been demonstrated for a variety of systems, such as metal nanohole arrays, nanoparticles, rods and dimers, combined with photochromic molecules, J-aggregates, and monolayers of $\mathrm{MoS}_{2}, \mathrm{WS}_{2}$ and $\mathrm{WSe}_{2}$. We discuss work on optical switching realized by changing photochromic molecules by UV and visible light between forms that couple strongly or weakly with the electromagnetic field mode of the plasmonic nanostructure. Other forms of optical control such as use of polarization and phase are briefly covered. We review research on electrical, thermal and chemical active control of light-matter coupling in plasmonic systems.
\end{abstract}




\section{Keywords}

Plasmonics, Strong coupling, Nanostructures, Quantum optics, Quantum emitters, Polaritons 
In strong coupling, an emitter interacts with electromagnetic field mode enabling coherent energy transfer between the two. This leads to hybridization of the energy levels of the emitter (for instance, a fluorescent molecule or a quantum dot) and the modes, and is manifested as anti-crossing of the original dispersion energies. The formation of energy gap in the dispersion of the hybrid modes is known as Rabi splitting. Plasmonic systems support modes that combine electron plasma oscillations together with electromagnetic radiative and near fields. This allows small mode volumes which is favourable for strong coupling. Although plasmonic structures are inherently lossy, strong coupling has been reached in various plasmonic systems ${ }^{1}$ by using high concentrations of fluorescent molecules having large oscillator strengths. Recently, strong coupling has been achieved for ever smaller amount of emitters. $^{2-4}$

Within the lossless coupled modes model, when two modes with energies $E_{1}$ and $E_{2}$ are coupled with a coupling energy $\Omega$, the new normal modes are shifted by the following amounts from the initial ones: $\pm\left(\left(E_{2}-E_{1}\right) / 2-\sqrt{\left(E_{1}-E_{2}\right)^{2} / 4+\Omega^{2} / 4}\right)$. This gives $\pm \Omega / 2$ at resonance and approximately $\pm \Omega^{2} /\left(4\left(E_{2}-E_{1}\right)\right)$ far away from it. Thus the energy shifts can be controlled by changing $\Omega$, or by changing the energy $E_{1}$ (or $E_{2}$ ) to tune the system in or out of resonance. While "active control of coupling" strictly speaking means active control of $\Omega$, in this review we have included also a few works which actively tune the resonance condition (change of $E_{1}$ or $E_{2}$ ) since it can be also viewed as a way to change the effect of the interaction on the mode energies.

Modern nanofabrication techniques allow engineering the size, shape, and material of plasmonic structures. Similarly the opportunities for designing the properties of the emitters are wide-ranging. Optimizing the structures and the positioning of the emitter to modify the coupling conditions constitutes a broad field of research. ${ }^{1,5-10}$ Tailoring the structures and the emitters can be thought as passive (or static) ways to control the plasmon-exciton coupling.

For further exploration and applications of the plasmon-exciton coupling, it is of interest 
to make it externally controllable during operation, in particular, switchable in ultrafast time-scales. In this review, we focus on active (or dynamic) ways to control coupling by external stimuli. Various types of organic materials and functional molecules have been harnessed to provide active control in plasmonics. ${ }^{11,12}$ We provide an overlook on the recent advances in active optical, electrical, thermal, and chemical control of strong coupling.

\section{Optical control of plasmon-exciton strong coupling}

Optical control of strong coupling in plasmonics has been established by using emitters whose optical properties can be tuned by the exciting field. Alternatively, the exciting field can be used to tune the resonance properties of the plasmonic structure.

Photoswitchable fluorophores enable all-optical controlling of optoelectronic devices. Photochromic molecules change their conformation and optical properties upon exposure to light of suitable wavelength. Majority of current applications use photochromic molecules as a part of a macroscopic optical component, such as a lense or sunglasses. In this case, a macroscopic number of molecules is used to obtain the desired functionality. However, there exists an increasing interest to use photochromic molecules in nanotechnology where their small physical size can be utilized. In plasmonic systems, the small size of photochromic molecules allows, in principle, them to be positioned to the hotspots of the plasmonic near field. This might enable, for instance, photochromic switching of strong coupling between a single molecule and a plasmonic nanostructure.

Photoswitchable strong coupling has been demonstrated in different systems supporting confined electromagnetic modes, such as optical cavity modes and surface plasmons,${ }^{13}$ hybrid plasmon-waveguide modes, ${ }^{14}$ and plasmon resonances in periodic nanoparti-

cle arrays. ${ }^{15}$ The capability of strong coupling, together with the reported ${ }^{16}$ ultrafast (1 ps) switching speeds, make photochromic molecules attractive candidates for optical memories and switches as well as for high density data storage. 
Reversible switching of ultrastrong coupling between photochromic molecules and an optical cavity was demonstrated by Schwartz et al. ${ }^{13}$ In the experiment, a cavity consisting of silver mirrors was filled with photochromic spiropyran-based molecules in a polymethyl methacrylate (PMMA) matrix. The photochromic transformation occurs between spiropyran (SP) and merocyanine (MC) forms of the molecule, see Fig. 1. Upon exposure to light at the ultraviolet (UV) regime, the molecules convert from SP form into MC form whose absorption peak $(2.2 \mathrm{eV})$ overlaps with the cavity mode resonance. As a result, a Rabi splitting of up to $700 \mathrm{meV}$ was observed. The splitting accounts for $32 \%$ of the exciton energy, confirming the ultrastrong coupling. The conversion between the two molecular forms is fully reversible by visible (green) light illumination. Here the reported time scale for photoconversion (10 min at $10 \mathrm{~mW} / \mathrm{cm}^{2}$ ) is likely limited by the light intensity used for the switching rather than the actual transition speed between two molecular conformations, which can be on the order of picoseconds. ${ }^{16}$

Compared to an optical cavity, a plasmonic structure offers more appealing features in terms of tunability, open access and nanoscale integration. However, plasmonic structures have high losses due to the metallic component. To compare the coupling strengths in these two systems for otherwise similar conditions, Schwartz et al. ${ }^{13}$ studied strong coupling also between the SP-based molecules and surface plasmon modes. The system consisted of a periodic hole array made of silver and coated with a PMMA film doped with the photochromic molecules. Similarly, as with the optical cavity system, a splitting was observed in the transmission spectrum upon converting the molecules from the SP to the MC form, see Fig. 2. The magnitude of the Rabi splitting was $650 \mathrm{meV}$ which is comparable to the value obtained in the optical cavity.

High ohmic losses of the plasmonic cavities set limitations for both the coupling strength and the capability for optical guiding and switching. Hybrid-plasmon waveguide modes (HPWMs) combine the plasmonic field enhancement with long-range propagation in a dielectric waveguide. Compared to conventional dielectric-based microcavities, incorporating 


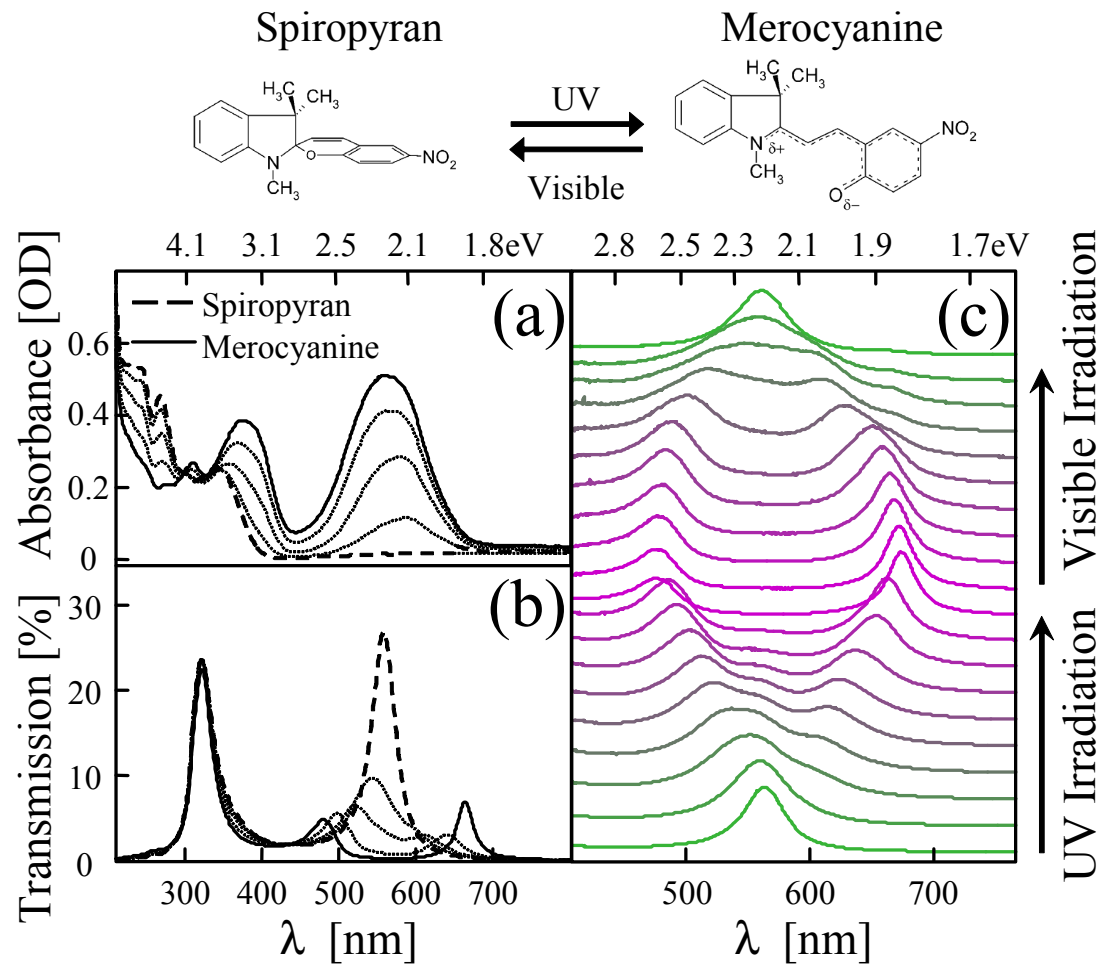

Figure 1: Spectral evolution of the photochromic molecules upon reversible switching. The photochromic molecule is indolinospiropyran (1',3'-dihydro-1',3',3'trimethyl-6-nitrospiro [2H-1-benzopyran-2,2'-(2H)-indole]). In its relaxed closed form, spiropyran (SP) is transparent in the visible region of the spectrum, whereas the open form merocyanine $(\mathrm{MC})$ absorbs strongly at around $2.2 \mathrm{eV}(560 \mathrm{~nm})$. The switching from the SP to the MC form is perfofmed by UV light, and reversed by visible (green) light illumination. (a) Absorption and (b) transmission spectra of the two forms of the photochromic molecules: spiropyran (dashed line) and merocyanine (solid line). (c) Spectral evolution of the transmission spectrum upon subsequent UV and visible light illumination. Reprinted with permission from Ref. 13. Copyright (2011) by the American Physical Society.

plasmonics to the cavity provides higher electric field enhancement and mode confinement. Lin et al. ${ }^{14}$ studied reversible switching of Rabi splitting in HPWMs in a system consisting of an array of Al nanodisks covered by a PMMA film containing the photochromic SP-based molecules. By alternative UV and visible light illumination, and therefore switching between the MC and SP forms of the molecules, a Rabi splitting of $572 \mathrm{meV}$ was reported. This accounts for about $25 \%$ of the exciton energy.

The reversibility of the Rabi splitting is apparent in Fig. 3(b). The transmission spectrum 


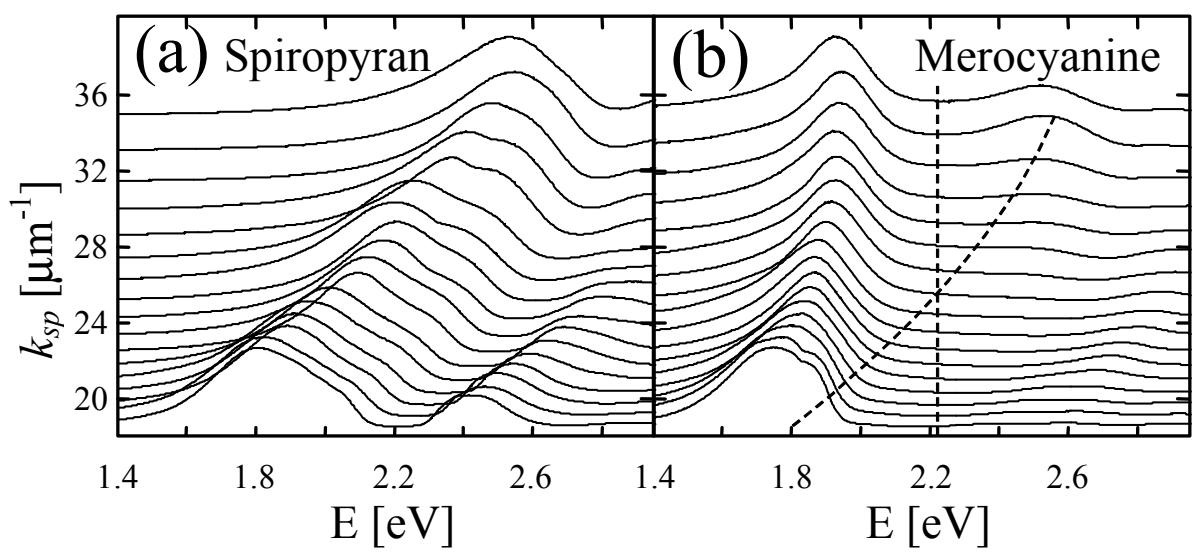

Figure 2: Dispersion of the surface-plasmon modes in a hole array before and after photoswitching. The photochromic molecules are in (a) spiropyran and in (b) merocyanine form, revealing splitting of $650 \mathrm{meV}$ between the lower and upper dispersion branch after photoswitching. The vertical dashed line in (b) shows the exciton energy, whereas the curved dashed line in (b) indicates the parabolic surface-plasmon mode dispersion from (a). Reprinted with permission from Ref. 13. Copyright (2011) by the American Physical Society.

of the sample before photoconversion ("As-prepared") shows initially a single resonance at the wavelength of $560 \mathrm{~nm}$, corresponding to the periodicity of the nanodisk array $(430 \mathrm{~nm})$. When the molecules are converted into the MC form by UV light illumination, the resonance dip is split into two new ones at wavelengths of 500 and $640 \mathrm{~nm}$. Recovering the molecules back to the SP form by green light illumination transforms the spectrum back to the initial single-resonance shape. The corresponding anti-crossing in the energy dispersion is shown in Fig. 3(a). The authors noted that the switchability of the SP molecules can show fatigue after performing multiple cycles of photoconversion. As an interesting follow-up to their experiments, Lin et al. ${ }^{14}$ applied photochromic switching of the resonant wavelengths of the HPWMs to creating optically rewritable waveguides.

Photoswitchable strong coupling was demonstrated in a plasmonic nanoparticle array by Baudrion et al. ${ }^{15}$ In the experiment, a silver nanoparticle array embedded in a spiropyrandoped PMMA film was illuminated by UV light, which yield to Rabi splitting of $294 \mathrm{meV}$ between the (near-field) plasmon resonance modes and the molecules. The smaller relative Rabi splitting (13\% of the exciton energy) compared to the experiments of Schwartz et 

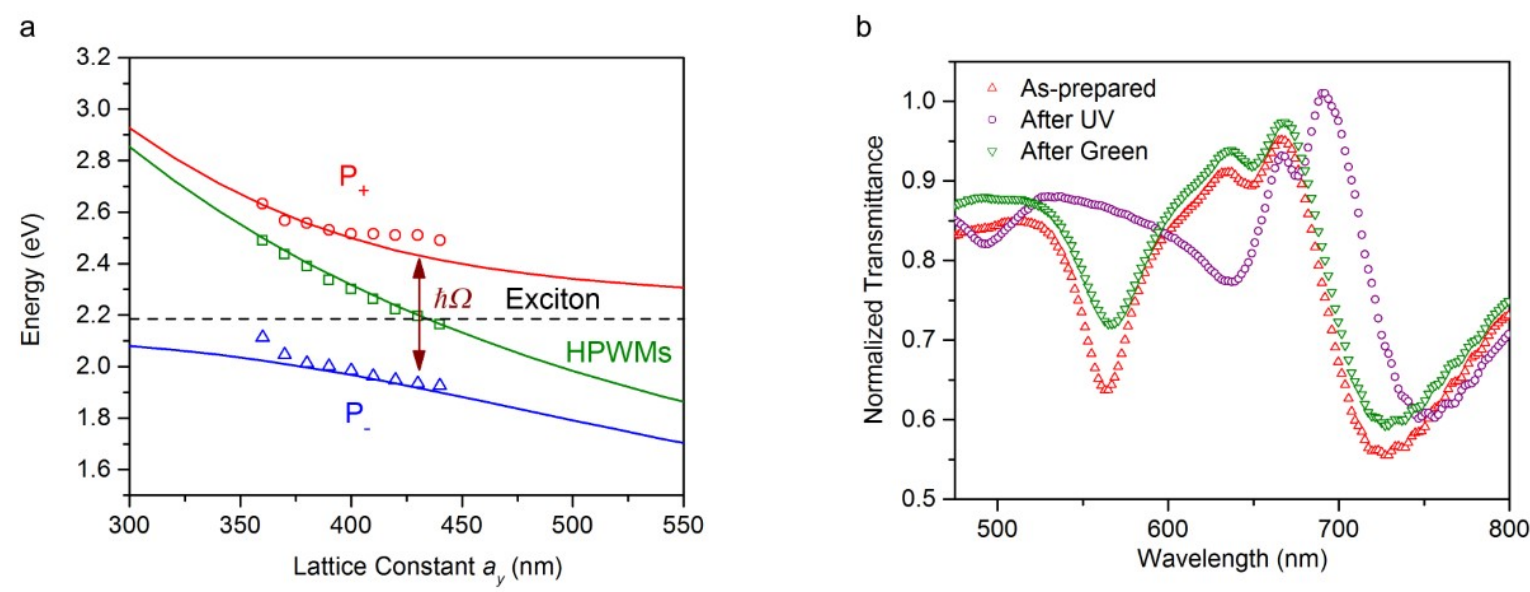

Figure 3: Photochromic switching of strong coupling in a system supporting hybrid-plasmon waveguide modes (HPWMs). (a) Dispersion of the HPWMs in a nanodisk array upon photoswitching. The green markers indicate the energies of the bare HPWMs, and the red and blue markers show the resulting energies after photoswitching. The solid lines are obtained by fitting a two-coupled-oscillators model into the measured data. The largest reversible splitting observed was $\Omega=572 \mathrm{meV}$. The horizontal dashed line indicates the molecular transition energy for the merocyanine form. (b) Transmission spectrum of the system in the initial state ("As-prepared"), after UV illumination, and after green light illumination. Reversibility of the Rabi splitting is clearly visible upon photoswitching. Reprinted with permission from Ref. 14. Copyright (2016) by the American Chemical Society.

al. ${ }^{13}(32 \%)$ was explained by the lower quality factor of the dipolar resonances compared to those of optical cavity or surface plasmons. Through a series of measurements varying the nanoparticle diameter between 70-110 nm (the height was fixed to $50 \mathrm{~nm}$ ), the authors concluded that the strongest anti-crossing was obtained having the plasmon resonance close to the absorption of the MC form, see Fig. 4(a). The observation was further confirmed by computational extinction spectrum displayed in Fig. 4(b). The reversible switching between the MC and SP forms of the molecules was performed by subsequent UV illumination and heating of the molecule film at $40{ }^{\circ} \mathrm{C}$ for 10 minutes. The thermal treatment caused visible fatigue in the polymer layer, suggesting that for serial cycling it would be better to use all-optical switching.

Reversible switches based on photochromic molecules have been created in several other plasmonic systems as well. For instance, in surface plasmon-polariton waveguides based 

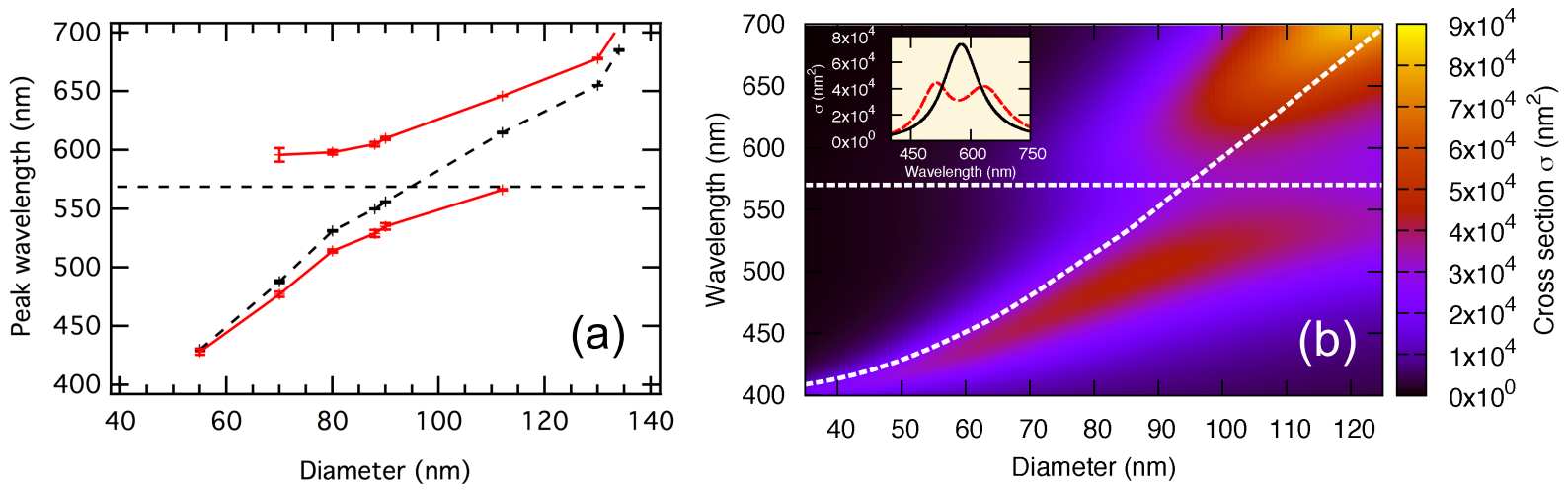

Figure 4: Dispersion of the plasmon resonance modes in a nanoparticle array upon photoswitching. (a) The position of extinction peak modes as a function of nanoparticle diameter. The dashed black line corresponds to the dispersion of plasmon resonances when the molecules are in the spiropyran form. The horizontal black line marks the absorption of the merocyanine (MC) form. Splitting of $294 \mathrm{meV}$ between the resulting lower and upper dispersion branches (red curves) is observed. (b) Computed spectrum of extinction cross section as a function of nanoparticle diameter. The cross section is calculated from the polarizability of a nanoparticle. ${ }^{15,17}$ The dashed white curve corresponds to the dispersion of plasmon resonances, and the horizontal line points out the MC absorption. The position in the map where maximum splitting occurs is shown in the inset (red dashed line). The inset also shows the absorption profile for the MC form (solid line). Adapted with permission from Ref. 15. Copyright (2013) by the American Chemical Society.

on periodic gratings on $\mathrm{Al}$ film ${ }^{18}$ and plasmon resonances in (disordered) arrays of gold nanospheres, ${ }^{19}$ nanorods, ${ }^{20}$ and nanodisks. ${ }^{21}$ The switchability of photochromic molecules can be also utilized in controlling lasing. Photochromic effect has been applied to all-optical tuning $^{22}$ and switching ${ }^{23}$ of lasing in photonic crystals.

Photoswitchable fluorophores are one example of materials harnessed for all-optical control of coupling in plasmonics. Other organic and inorganic materials that have been used include, for example, J-aggregates, functional polymers, and semiconductor monolayers. ${ }^{11}$ J-aggregates are a type of organic dye molecules that undergo red-shifting and spectral narrowing of absorption spectrum when concentration exceeds a certain limit. The mechanism is explained by the collective response of molecular domains that are formed when tens of molecules couple optically. ${ }^{24,25}$

All-optical control is not always targeted to changing the properties of the emitters, but 
rather manipulating the electric field and resonances of the plasmonic structure, which then affects the coupling strength. Wavelength is just one of the numerous variables of the exciting field that can be tuned when pursuing all-optical control. Other reported approaches include, for instance, changing the polarization, pump fluence, and incident angle.

Polarization-controlled strong coupling between J-aggregates and localized surface plasmon resonance (LSPR) modes in single dimers was demonstrated by Schlather et al. ${ }^{26}$ Placing two gold nanodisks close (15 nm gap) to each other creates a field hot spot in the middle of the dimer, hence enhancing the interaction between the LSPRs and the J-aggregate excitons. The dimer supports two SPR modes (longitudinal and transverse polarization) from which the scattered light can be observed independently. The dimensions of the dimers (diameters 60-115 $\mathrm{nm}$ ) were chosen so that their longitudinal resonance mode matched with the J-aggregate exciton energy $(1.79 \mathrm{eV})$. Strong coupling was observed between the dimers and excitons for the longitudinal SPR, whereas barely no coupling was observed when exciting the transverse SPR mode, see Fig. 5(a)-(b). The effect of polarization on the coupling was directly attributed to the decrease of nearfield enhancement, see Fig. 5(c)-(e). The coupling strength was gradually tuned by changing the polarization of the exciting field with small steps. The highest measured Rabi splittings were in the range of $230-400 \mathrm{meV}$ (13-22\% of the exciton energy).

Zhang et al. ${ }^{27}$ studied strong coupling between J-aggregates and SPPs on silver hole (aperture) arrays. The periodicities of the two-dimensional array were different in orthogonal directions, and therefore the polariton modes arising from strong coupling were dependent on the polarization of the exciting field. Both transverse-electric (TE) and transverse-magnetic (TM) field polarizations yielded to strong coupling with observed splitting energies of 200 $\mathrm{meV}(9 \%)$ and $250 \mathrm{meV}$ (12\% of the exciton energy), respectively. Interestingly, the two polarizations were found to produce polariton bands with opposite directions of propagation, see Fig. 6. The TE-polarized exciting field created polariton bands that disperse towards higher energies with increasing incident angle. In contrast, the TM-polarized field caused 

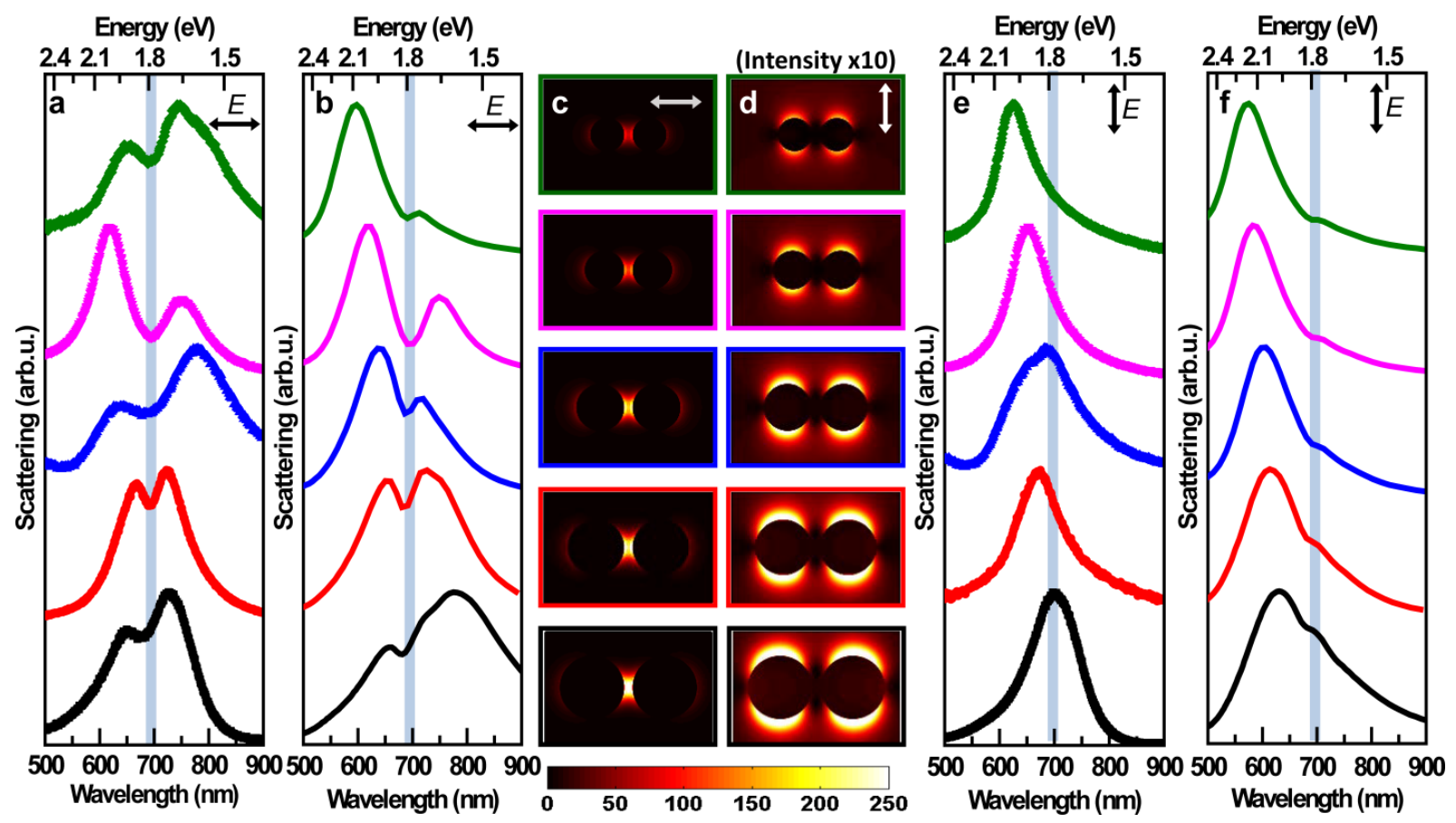

Figure 5: Polarization-dependent control of the coupling on a single plasmonic dimer structure accompanied with J-aggregates. (a) Measured and (b) computed (FDTD) scattering spectra for the system with different polarization angles. Splitting to lower (LB) and upper (UB) polariton bands becomes apparent as the polarization angle decreases (polarization changes from transverse to longitudinal). Also red shift of UB peak is visible upon changing the polarization. (c) Near-field profiles for the dimer with different polarization directions (white arrows). (d) Spectral position of the UB peak as a function of the polarization angle. (e) Scattering intensity of the LB peak as a function of polarization angle. The blue solid line in both (d) and (e) shows the computed polarization-dependence of the nearfield enhancement in the gap. Adapted from Ref. 26. Copyright (2013) by the American Chemical Society.

polariton bands dispersing towards lower energies when the incident angle increases. Thus, by simply changing the polarization of the exciting field one could switch the direction where the polariton energies propagate on the array.

Polarization can be used also in the opposite way than for controlling the strong coupling. Namely, the polarization of observed photoluminescence has been used as a measure of the contribution of the exciton part in strongly coupled modes between SPPs and dye molecules. ${ }^{28}$

Pump-controlled strong coupling between SPPs on a gold nanoslit array and cyanine J-aggregates layered on top of the array was demonstrated by Vasa et al. ${ }^{25}$ A $55 \mathrm{meV}$ 
(a)

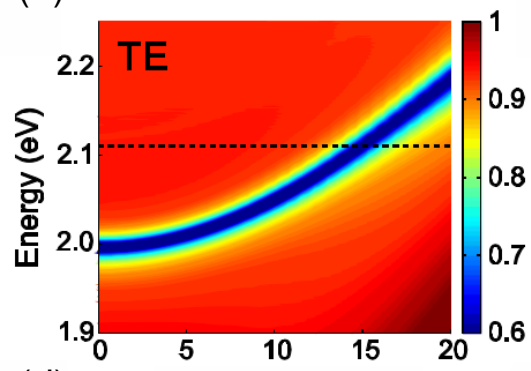

(d)

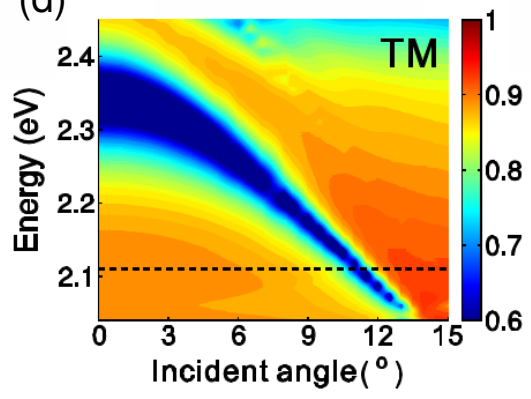

(b)

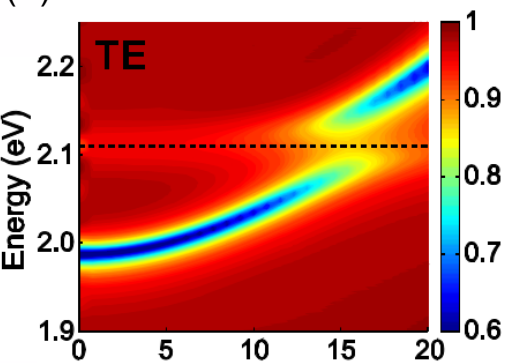

(e)

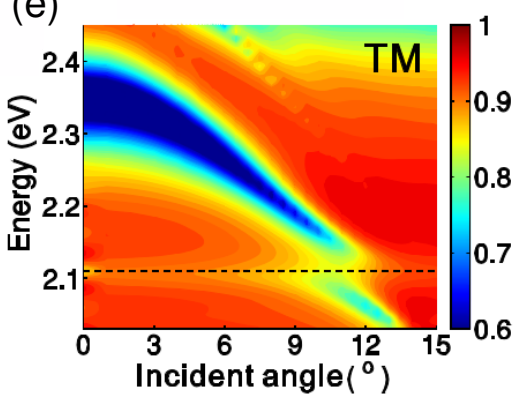

(c)

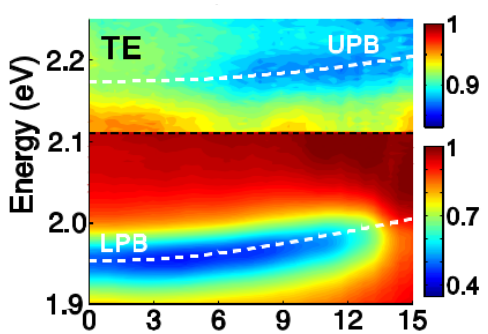

(f)

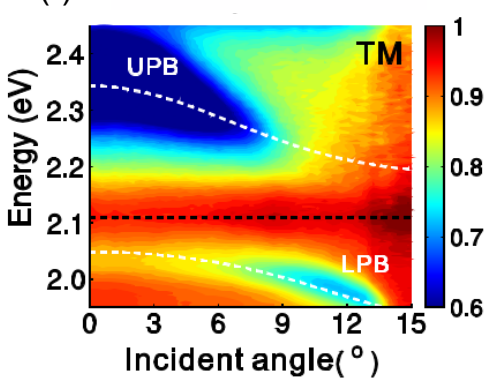

Figure 6: Dispersion of the plasmon resonance modes in a hole array covered with J-aggregates. Calculated reflection spectrum under TE-polarized exciting field for (a) bare array and (b) array covered with J-aggregates. (c) Measured reflection spectrum under TE-illumination for the array covered with J-aggregates. Splitting of the dispersion into lower (LBP) and upper polariton band (UBP) is observed. (d)-(f) show the respective results for TM polarization. Adapted from Ref. 27. Copyright (2017) by the Optical Society of America.

splitting was reversibly switched on and off in sub-picosecond timescale by modulating the pump fluence. The system was excited by ultrashort ( $80 \mathrm{fs}$ ) pulses, followed by a similar but lower intensity probe pulse. The off-resonant pump induces saturation effects in the exciton absorption, which effectively reduces the total oscillator strength. Different incident angles were used for tuning the SPPs on the array to achieve optimal overlap of the SPP resonance and the J-aggregate exciton energy $(1.78 \mathrm{eV})$. The experiment was conducted under vacuum at temperature of $77 \mathrm{~K}$ to minimize photobleaching of the cyanine dye. The value of the observed Rabi splitting was relatively small (3\% of the exciton energy) compared to previously reported strong coupling experiments with J-aggregates. This was attributed to thinner dye film $(50 \mathrm{~nm})$ and narrower slit grating (45 $\mathrm{nm}$ slit width, 380-430 nm period) chosen to increase the polariton lifetimes. 
As a follow-up to this work, Vasa et al. ${ }^{29}$ reported real-time observation of sub-picosecond Rabi oscillations (with $110 \mathrm{meV}$ splitting). In this work, even shorter (15 fs) pulses were used for excitation. To avoid photobleaching the experiments were conducted under vacuum, but at room temperature.

Monolayers of transition metal dichalcogenides (TMDCs) are semiconductor atomic layers with tunable optical properties. TMDCs are promising candidates for actively controlled plasmonic devices due to electronic structure with a direct bandgap and tightly bound excitons. ${ }^{30}$ For example, molybdenum disulfide $\left(\mathrm{MoS}_{2}\right)$ monolayer has been combined with a plasmonic structure to enable enhanced photoluminescence ${ }^{31}$ and strong coupling. ${ }^{32}$ Also all-optical control of the coupling has been demonstrated in such hybrid system. ${ }^{33}$

Li et al. ${ }^{34}$ studied all-optical control of the exciton binding energy in $\mathrm{MoS}_{2}$ monolayer accompanied with nanoparticles. Control of coupling was not considered in this work, but the results formed a basis for later studies of coupling control. The exciton binding energy is dependent on the $\mathrm{MoS}_{2}$ doping density, which changes when hot electrons are transferred from plasmons into the monolayer. Exciting the LSPRs of the gold nanoparticles caused redshifting of the absorption and emission spectra of the monolayer. In addition, the overall absorption intensity was found to decrease upon increasing either the pump power or the nanoparticle concentration.

The work Li et al. ${ }^{34}$ was extended by $\mathrm{Zu}$ et $a l .{ }^{33}$ to control the coupling. The features of the $\mathrm{MoS}_{2}$ monolayer were deployed for controlling the coupling between the monolayer and a silver nanodisk array. The dielectric permittivity of the monolayer was changed by generating excitons. Due to the change in the dielectric environment, the exciton absorption peak red-shifted with respect to the surface plasmon resonance when the power of the exciting field was increased, see Fig. 7. Again, also the overall intensity of absorption decreased by increasing pump power, which is interpreted as enhanced plasmon - exciton coupling. The excitons generated in the monolayer increase the total oscillator strength. As an additional experiment, $\mathrm{Zu}$ et al. showed that using silver nanorods instead of symmetric disks allowed 
switching between weak and strong coupling by simply changing the polarization of the exciting field. The experiments were conducted at room temperature and in the visible wavelength regime.
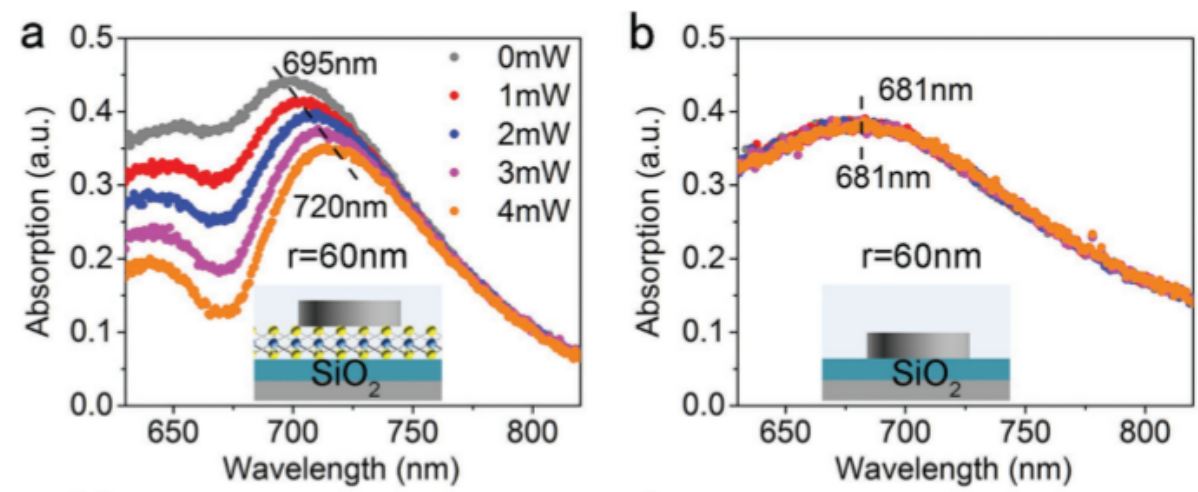

Figure 7: Absorption spectra of the silver nanodisk array - $\mathrm{MoS}_{2}$ monolayer hybrid system. (a) Absorption spectra of the nanodisk array fabricated on the $\mathrm{MoS}_{2}$ monolayer as a function of pump power. The absorption spectra shows red shift of the peak from $720 \mathrm{~nm}$ to $695 \mathrm{~nm}$, when the pump power is increased from 0 to $4 \mathrm{~mW}$. Also the overall absorption intensity shows decrease upon increasing the pump power. (b) Absorption spectra of the nanodisk array remains unaltered upon increasing the pump power. The radii of the disks was $60 \mathrm{~nm}$. Silicon dioxide $\left(\mathrm{SiO}_{2}\right)$ and silver films are used as the base substrate. Adapted from Ref. 33. Copyright (2016) by Wiley-VCH Verlag GmbH \& Co. KGaA.

Other ways to all-optical control of coupling include, for example, varying the phase of the exciting field. Sukharev et al. ${ }^{35}$ proposed using phase-modulated (chirped) laser pulses to control which exciton transitions are allowed in strongly coupled hybrid modes between SPPs and quantum emitters. Demetriadou et al. ${ }^{36}$ showed by simulations that a metal nanorod dimer exhibits even (bright) and odd (dark) modes that can be selectively excited by utilizing the parity symmetry of the structure, for instance by illuminating the structure from two sides with light fields of either zero or $\pi$ phase difference. The even and odd modes were found to have equal coupling to a molecular layer, but due to their different damping rates, the Rabi splittings were different. The phase of the incident light could therefore be used to control the Rabi splitting. 


\section{Electrical control of plasmon-exciton strong coupling}

Electrical control of the plasmon-exciton coupling can be achieved by combining a plasmonic structure with a material whose optical or conductivity properties can be tuned by electricity. Such materials include, for instance, semiconductor (TMDC) monolayers, liquid crystals, and graphene.

Applying voltage to a monolayer has recently gained wide interest in the research on electrical control of plasmon-exciton coupling. ${ }^{37-41}$ The method is based on controlling the density of excitons in a monolayer by electrostatic doping.

Reversible electrical switching of strong coupling between a $\mathrm{MoS}_{2}$ monolayer and surface lattice resonances on a silver nanodisk array was demonstrated by Lee et al. ${ }^{38}$ The monolayer and the nanodisk array were fabricated in a field-effect transistor (FET) configuration which allows for injecting or ejecting charge carriers to the monolayer by applying voltage to the electrical contacts. Schematic of the system is presented in Fig. 8(a). Increasing concentration of charge carriers reduces the exciton oscillator strength (due to exciton bleaching). Therefore, altering the voltage between $0 \mathrm{~V}$ to $80 \mathrm{~V}$ induced switching of the plasmon-exciton $\left(\mathrm{A}^{\circ}\right)$ coupling from $63 \mathrm{meV}$ (3\% of the exciton energy) to zero splitting, see Fig. 8(b). The experiment was carried out at temperature of $77 \mathrm{~K}$. Interestingly, at low positive gate voltages the strong coupling was observed to the neutral excitons, whereas at higher positive gate voltages to the charged excitons (trions, $\mathrm{A}^{-}$), that is fermionic excitonplasmon polaritons. This lead to the formation of distinct polariton bands for excitons and trions. The maximum splitting observed for plasmon-trion coupling was $42 \mathrm{meV}$ (2\% of the trion energy), see Fig. 8(b). Switching between bosonic and fermionic polariton states was achieved simply by altering the gate voltage, which makes the system interesting for experiments with the fermionic polariton states.

Also Li et al. ${ }^{39}$ studied electrical control of coupling between a $\mathrm{MoS}_{2}$ monolayer and a single gold nanodisk. The experiment was done in a similar FET configuration but at room temperature. The voltage over the monolayer was switched between $\pm 8 \mathrm{~V}$. The switching 
(a)

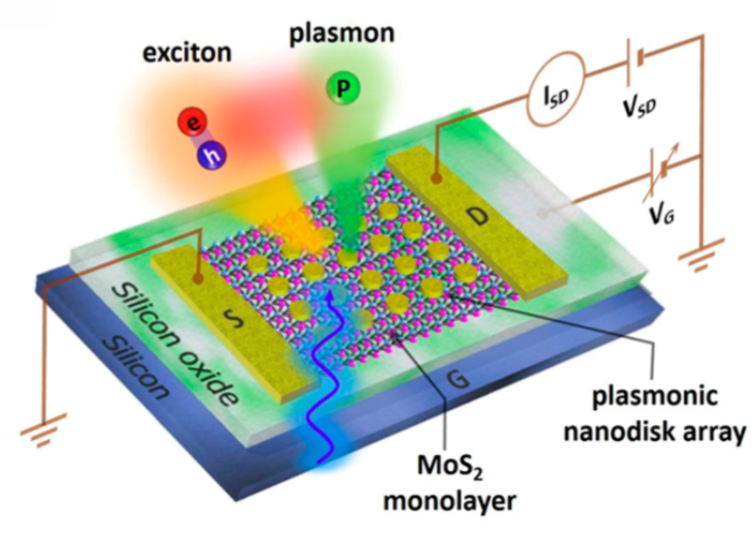

(b)

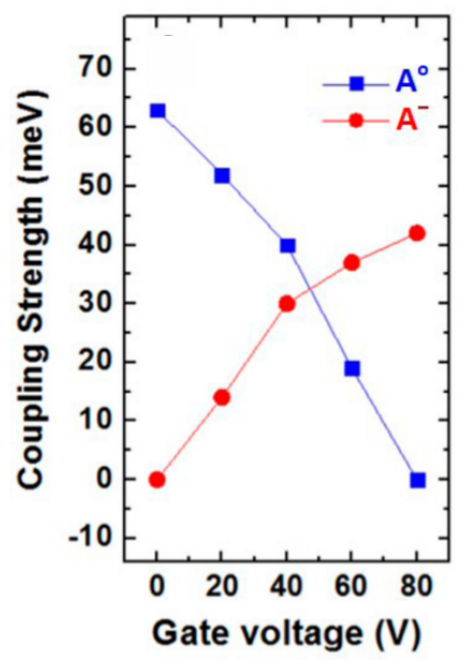

Figure 8: Electrical control of coupling in a silver nanodisk array on a $\mathrm{MoS}_{2}$ monolayer. (a) Schematic of the system: $\mathrm{MoS}_{2}$ monolayer and silver nanodisk array fabricated in a field-effect transistor (FET) configuration which allows for injecting and ejecting charge carriers to the monolayer by applying voltage on the electrical contacts. (b) Coupling strength (obtained by coupled oscillator model fitting) for excitons $\left(\mathrm{A}^{\circ}\right)$ and trions $\left(\mathrm{A}^{-}-\right)$ as a function of gate voltage. Adapted from Ref. 38. Copyright (2017) by the American Chemical Society.

time was reported to be less than $200 \mathrm{~ms}$, but this estimation was strictly limited by the integration time of the spectrometer. The authors estimate that the switching practically occurs in nanosecond scale.

Tungsten disulfide $\left(\mathrm{WS}_{2}\right)$ monolayers possess similar features than the $\mathrm{MoS}_{2}$. Wen et $a l .{ }^{41}$ demonstrated electrical control of strong coupling between a single gold nanorod and a $\mathrm{WS}_{2}$ monolayer in a FET geometry. The nanorod interacts with the monolayer through only a few (5-18) excitons, the process thus approaches the quantum limit. Whereas applying positive gate voltage did not have effect on the photoluminescence intensity and $\mathrm{WS}_{2}$ exciton transition, a negative one caused suppression and redshift of the photoluminescence. In addition, a low-energy shoulder appeared in the photoluminescence spectrum when negative voltage was applied. This is attributed to the formation of a trion band similarly as reported with the $\mathrm{MoS}_{2}$ monolayer by Lee et al. ${ }^{38}$ and Li et al. ${ }^{39}$ Strong coupling between the LSPR modes and excitons could be switched on and off by switching the bias voltage between 0 
and $-120 \mathrm{~V}$. The observed Rabi splittings were around $91-133 \mathrm{meV}$ (5-7\% of the exciton energy). The spectra were measured one second after switching the voltage to make sure the FET device had stabilized. Thus the actual switching time could not be deduced from this experiment.

Other types of electrical control of coupling, based on tuning the resonance properties of the plasmonic structures, have been also studied. Liquid crystals (LCs) are a promising group of materials for active plasmonics, as their properties can be modulated through electrically, optically, or thermally induced phase transition. The LCs enable creating plasmonic switches with low applied voltages. ${ }^{42,43}$ The LCs have been used to tune the surface plasmon resonances, for instance in gold nanodot arrays, ${ }^{44}$ hole arrays, ${ }^{45}$ and nanorods. ${ }^{42,46}$ Chung-Tse et al. ${ }^{47}$ harnessed the tunability of LCs for controlling coupling between quantum emitters (dots) and LSPR modes in gold nanoparticles. Applying voltage $(0-8 \mathrm{~V})$ changed the dielectric permittivity of the LCs, which shifted the plasmon resonance. Consequently resonance condition is modified and the effect of the coupling on the system energies is changed. A comprehensive review on active plasmonics enabled by LCs is given in Ref. 48.

One approach to control coupling between nanostructures and emitters is to sink the system into an electrolyte solution, as exemplified by Minamimoto et al. ${ }^{49}$ The system consisted of gold nanoparticles embedded to a conductive substrate (acting as the electrode), covered with dye molecules (HITC). Low voltage $(0 \ldots-0.8 \mathrm{~V})$ was applied to create an electrochemical potential which reduces the number of optically active HITC molecules, reducing the coupling strength. The observed Rabi splitting values were in the range of $0 \ldots 250 \mathrm{meV}$ (15 $\%$ of the exciton energy). The timescale for stabilization of the electrochemical potential was reported to be a few hundred seconds.

Metallic nanostructures enable also electromechanical control of the resonant properties. Miyata et al. ${ }^{50}$ applied voltage over a suspended gold nanowire placed on top of a gold thin film. Applying the voltage changed the gap between the nanowire and the film, thus enabling 
to dynamically tune the plasmonic resonances of the nanowire. Note that mechanical tuning of the plasmonic resonances can be realized also by simply deforming the substrate of the nanostructure. For example, Bedogni et al. ${ }^{51}$ fabricated gold nanoparticle arrays on a flexible (PDMS) film, and tuned the surface lattice resonances by reversibly stretching the film which changed the the array periodicity.

\section{Thermal control of plasmon-exciton strong coupling}

Thermal control of plasmon-exciton coupling can be established by combining the plasmonic structure with a material that changes its optical or conductivity properties with varying temperature. For example, LCs with temperature-tunable refractive index have been used to shift the surface plasmon polariton resonances on a gold hole array. ${ }^{52}$ Also thermally tunable functional polymers have been used to switching of the surface plasmon resonances in gold nanoparticles. ${ }^{53}$

In the previous section, we described the study of Wen et al., ${ }^{41}$ where a $\mathrm{WS}_{2}$ monolayer was used to control strong coupling electrically. In the same study, also temperature scanning was used to control strong coupling between the $\mathrm{WS}_{2}$ monolayer and a single gold nanorod. The $\mathrm{WS}_{2}$ exciton is spectrally sensitive to the temperature variation. The photoluminescence spectrum of $\mathrm{WS}_{2}$ showed red shift of around $0.5 \mathrm{meV} / \mathrm{K}$ when heated, whereas the gold nanorod remained spectrally unaltered upon changing the temperature, see $9(\mathrm{a})-(\mathrm{b})$. This can be used to tune the resonance condition, see the scattering spectra measured at different temperatures, 9(c). Reversible Rabi splitting of $110 \mathrm{meV}$ (6\% of the exciton energy) was observed by changing the temperature in the range of 293-433 K. The experiment was conducted with steps of $20 \mathrm{~K}$, and it took roughly five minutes to stabilize the temperature at each step.

Switching of surface plasmon resonances has been demonstrated by controlling the heatinduced insulator-metal phase transition of vanadium dioxide $\left(\mathrm{VO}_{2}\right)$. This reversible change 
(a)
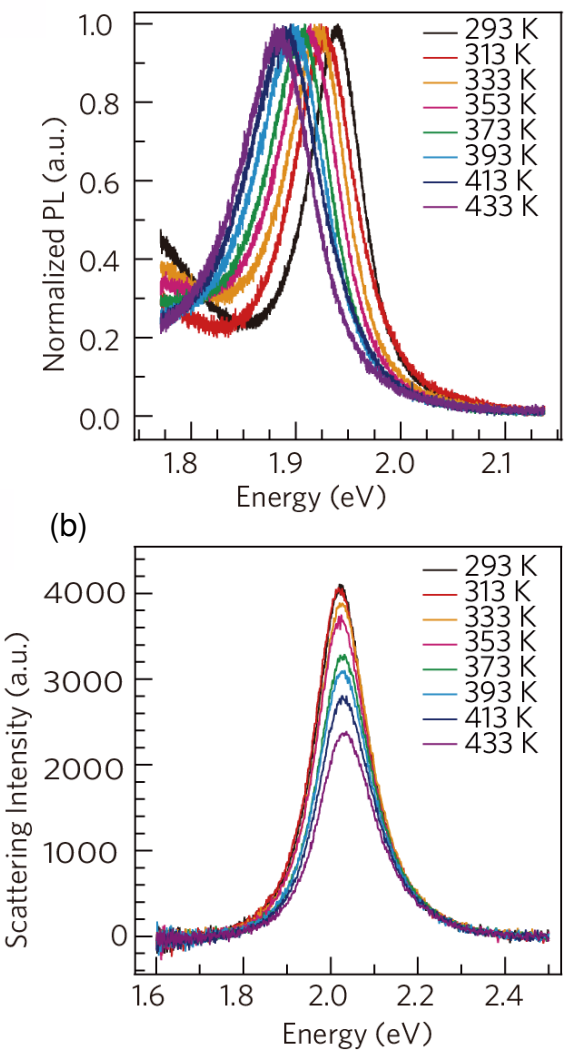

(c)

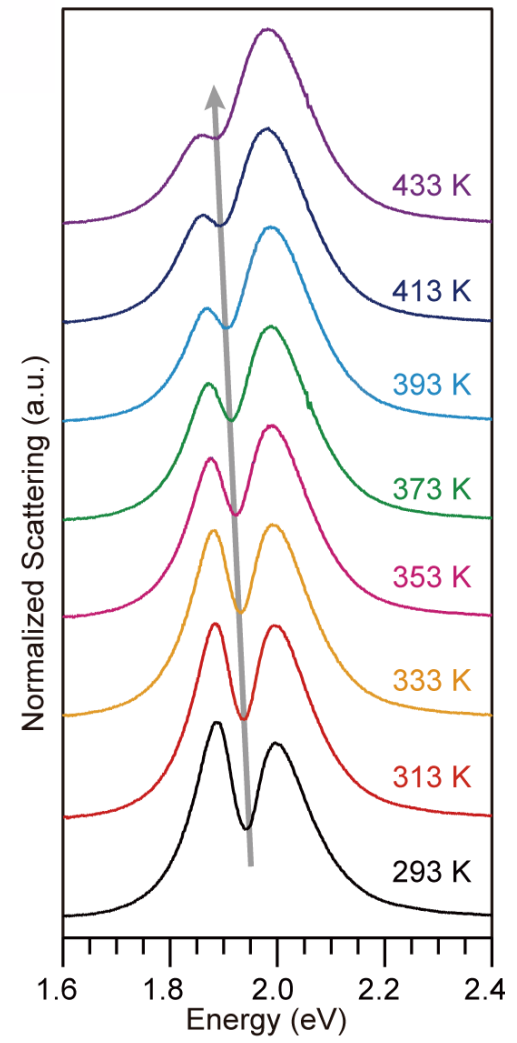

Figure 9: Thermal control of the interaction between the $\mathbf{W S}_{2}$ monolayer and a single gold nanorod Photoluminescence spectrum of (a) $\mathrm{WS}_{2}$ monolayer and (b) individual gold nanorod on a $\mathrm{SiO}_{2}$ substrate for temperatures between 293-433 K. (c) Scattering spectra of a $\mathrm{WS}_{2}$ monolayer - gold nanorod hybrid system. The exciton energy shows redshift (the gray arrow) upon increasing temperature which can be used for tuning the exciton and the nanorod energies in and out of resonance. At room temperature a Rabi splitting of about $110 \mathrm{meV}$ is observed. Adapted from Ref. 41. Copyright (2017) by the American Chemical Society.

of refractive index has been used to switch the plasmon resonances in, for instance, gold thin film accompanied by $\mathrm{VO}_{2}$ nanocrystals, ${ }^{54} \mathrm{Ag}-\mathrm{VO}_{2}$ bilayers, ${ }^{55}$ and gold nanorods on $\mathrm{VO}_{2}$ thin film. ${ }^{56}$ Note that in the metal phase the $\mathrm{VO}_{2}$ supports surface plasmon resonances itself. ${ }^{57}$ The phase transition occurs at critical temperature around $340 \mathrm{~K}$. The phase transition in $\mathrm{VO}_{2}$ can be induced also optically ${ }^{58,59}$ or by mechanical strain. ${ }^{60,61}$ 


\section{Chemical control of plasmon-exciton strong coupling}

Coupling between a plasmonic structure and excitons can be modified by changing the chemical composition of the system, which modifies the optical environment. Chemical control can be targeted to altering the properties of either the emitters ${ }^{62}$ or the plasmonic structure. ${ }^{63}$

Berrier et al. ${ }^{62}$ used nitrogen dioxide $\left(\mathrm{NO}_{2}\right)$ gas flow to control strong coupling between organic dye molecules (porphyrin) and surface plasmon polaritons (SPPs) on gold thin film. Exposure to nitrogen dioxide $\left(\mathrm{NO}_{2}\right)$ gas modifies the total oscillator strength of the porphyrin layer and generates exciton resonance $(1.77 \mathrm{eV})$. The oscillator strength affects directly the strength of coupling between the porphyrin and the SPPs, therefore a crossover from weak to strong coupling was seen by increasing the gas concentration, see Fig. 10. Splitting of the polariton modes was observed two minutes after starting the gas flow, and the highest observed Rabi splitting was $130 \mathrm{meV}$ (7\% of the exciton energy) with the range of gas concentrations used. The process could be reversed by heating the sample under nitrogen atmosphere.

Tungsten diselenide $\left(\mathrm{WSe}_{2}\right)$ monolayer is another TMDC material which has been shown to provide high photoluminescence enhancement when accompanied with a plasmonic structure. ${ }^{64}$ Zheng et al. ${ }^{63}$ studied strong coupling between a $\mathrm{WSe}_{2}$ monolayer and an individual silver nanorod residing on top of the monolayer. In the experiment, the LSPR of the silver nanorod was modified by depositing a dielectric (aluminium oxide) layer on top of the sample. As a transparent dielectric, aluminium oxide does not affect the $\mathrm{WSe}_{2}$ exciton energy, but causes a dielectric screening effect on the plasmonic structure. Typically this is seen as a red shift of the surface plasmon resonance, and the magnitude of the shift depends on the layer thickness. Note, however, that increasing oxide thickness can also cause a blue shift of the plasmon resonance, as was observed in a system consisting of a gold nanoparticle on oxidized $\mathrm{Al}$ substrate. ${ }^{65}$ Continuing the layer deposition until the LSPR overlapped spectrally with the $\mathrm{WSe}_{2}$ exciton yielded to Rabi splitting of $49.5 \mathrm{meV}$ (3\% of the exciton 


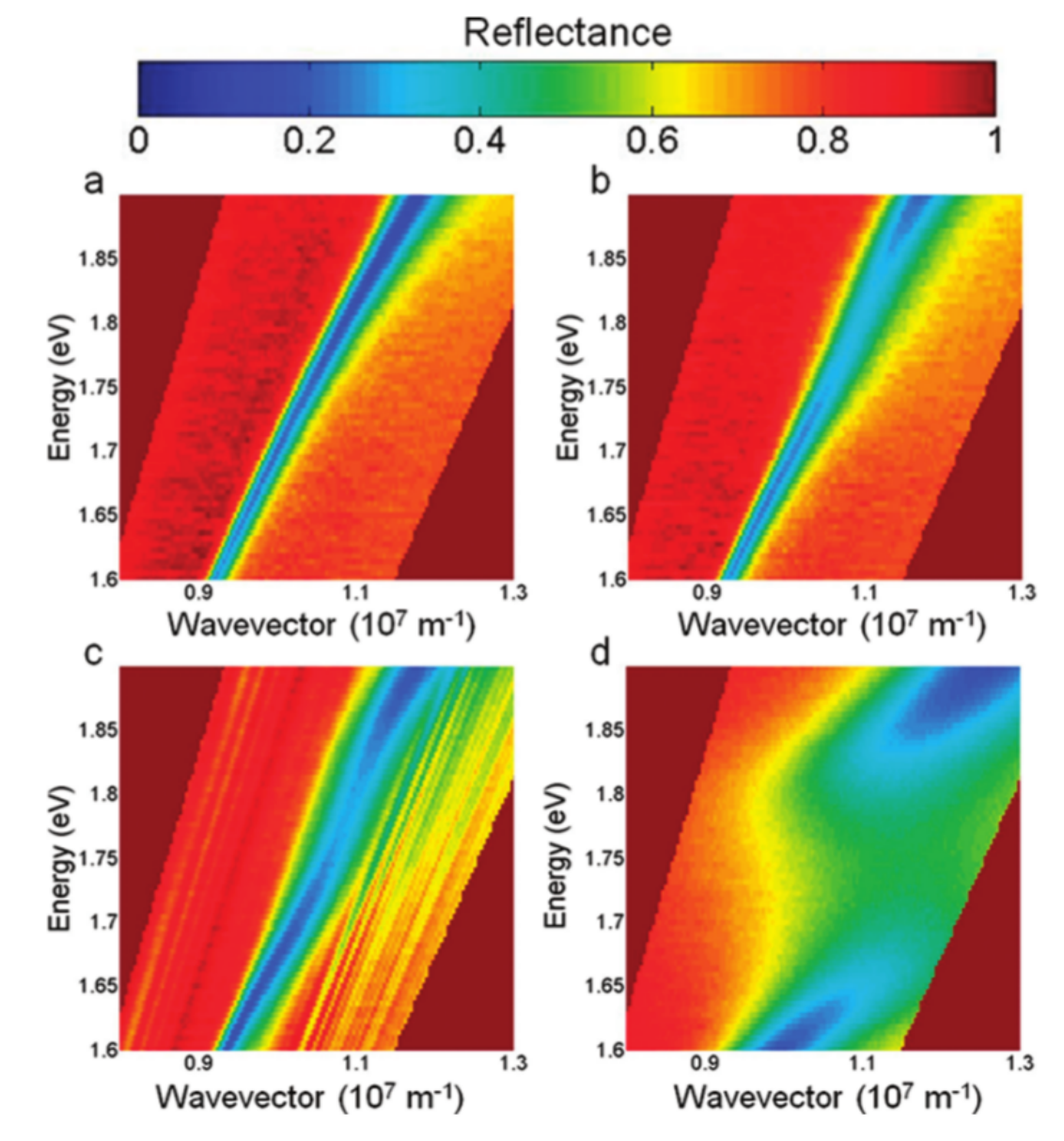

Reflectance

Figure 10: Chemical control of strong coupling between organic dye molecules and surface plasmon polaritons on gold thin film. Dispersion relations for different $\mathrm{NO}_{2}$ gas concentrations: (a) 0 parts per million (ppm), (b) $0.3 \mathrm{ppm}$, (c) $4.6 \mathrm{ppm}$, and (d) $6 \mathrm{ppm}$. Upon increasing the concentration, Rabi splitting up to about $130 \mathrm{meV}$ becomes apparent. Adapted from Ref. 62. Copyright (2011) by the American Chemical Society.

energy). Controlling the coupling by layer deposition is hardly an active control approach, since the process is irreversible, furthermore, this is control of the resonance condition, not of the coupling energy.

\section{Summary}

After achieving strong coupling in plasmonic systems, developing active control of the phenomenon is an important avenue of future research. To lay a basis for further efforts in this direction, we have reviewed here works that have emerged in recent years on active control 
of strong plasmon-exciton coupling.

All-optical control of plasmon-exciton coupling has been established by manipulating the optical properties of emitters, or alternatively by changing the field and resonance of the plasmonic structures. Switching by optical fields is an attractive option due to its fast, even ultrafast character. The use of the spiropyran and merocyanine forms of the indolinospiropyran molecule has turned out to be a feasible choice for photoswitching of strong coupling. All-optical control can be realized also by other means, e.g. by modulating the polarization of the exciting field.

In addition to optical control, we have discussed literature on electrical, thermal, and chemical control of strong coupling. Active control methods often deploy 2D materials like graphene or transition metal dichalcogenide (TMD) monolayers with properties that can be tuned by various ways optically, electronically, and thermally. Electrical control has been successfully demonstrated by applying voltage to a monolayer combined with a plasmonic structure, fabricated in a field-effect transistor (FET) configuration. Modulating light-matter coupling by voltage also paves the way towards electrically driven plasmonic devices.

Thermal and chemical control methods increase the diversity of options even further. Whereas harnessing thermal and chemical reactions for controlling plasmon-exciton coupling may lead to unprecedented applications, optical and electrical control provide currently the most promising solutions for ultrafast plasmonic switches and optoelectronic devices.

\section{Acknowledgement}

This work was supported by the Academy of Finland through its Centres of Excellence Programme (2012-2017) and under project nos. 284621, 303351, and 307419, and by the European Research Council (ERC-2013-AdG-340748-CODE). 


\section{References}

(1) Törmä, P.; Barnes, W. L. Strong Coupling Between Surface Plasmon Polaritons and Emitters: a Review. Reports on Progress in Physics 2015, 78, 013901.

(2) Zengin, G.; Wersäll, M.; Nilsson, S.; Antosiewicz, T. J.; Käll, M.; Shegai, T. Realizing Strong Light-Matter Interactions Between Single-Nanoparticle Plasmons and Molecular Excitons at Ambient Conditions. Phys. Rev. Lett. 2015, 114, 157401.

(3) Chikkaraddy, R.; de Nijs, B.; Benz, F.; Barrow, S. J.; Scherman, O. A.; Rosta, E.; Demetriadou, A.; Fox, P.; Hess, O.; Baumberg, J. J. Single-Molecule Strong Coupling at Room Temperature in Plasmonic Nanocavities. Nature 2016, 535, 127-130.

(4) Santhosh, K.; Bitton, O.; Chuntonov, L.; Haran, G. Vacuum Rabi Splitting in a Plasmonic Cavity at the Single Quantum Emitter Limit. Nature Communications 2016, 7.

(5) Kravtsov, V.; Berweger, S.; Atkin, J. M.; Raschke, M. B. Control of Plasmon Emission and Dynamics at the Transition from Classical to Quantum Coupling. Nano Letters 2014, 14, 5270-5275.

(6) Nugroho, B. S.; Malyshev, V. A.; Knoester, J. Tailoring Optical Response of a Hybrid Comprising a Quantum Dimer Emitter Strongly Coupled to a Metallic Nanoparticle. Phys. Rev. B 2015, 92, 165432.

(7) Piccione, B.; Aspetti, C. O.; Cho, C.-H.; Agarwal, R. Tailoring Light-Matter Coupling in Semiconductor and Hybrid-Plasmonic Nanowires. Rep Prog Phys 2014, 77.

(8) Eizner, E.; Avayu, O.; Ditcovski, R.; Ellenbogen, T. Aluminum Nanoantenna Complexes for Strong Coupling between Excitons and Localized Surface Plasmons. Nano Letters 2015, 15, 6215-6221. 
(9) Nagasawa, F.; Takase, M.; Murakoshi, K. Raman Enhancement via Polariton States Produced by Strong Coupling between a Localized Surface Plasmon and Dye Excitons at Metal Nanogaps. The Journal of Physical Chemistry Letters 2014, 5, 14-19.

(10) Zhang, Y.; Meng, Q.-S.; Zhang, L.; Luo, Y.; Yu, Y.-J.; Yang, B.; Zhang, Y.; Esteban, R.; Aizpurua, J.; Luo, Y.; Yang, J.-L.; Dong, Z.-C.; Hou, J. G. Sub-nanometre Control of the Coherent Interaction Between a Single Molecule and a Plasmonic Nanocavity. Nature 2017,8 .

(11) Chen, K.; Leong, E. S. P.; Rukavina, M.; Nagao, T.; Liu, Y. J.; Zheng, Y. Active Molecular Plasmonics: Tuning Surface Plasmon Resonances by Exploiting Molecular Dimensions. Nanophotonics 2015, 4, 186-197.

(12) Zayats, A. V.; Maier, S. Active Plasmonics and Tuneable Plasmonic Metamaterials; Wiley, 2013.

(13) Schwartz, T.; Hutchison, J. A.; Genet, C.; Ebbesen, T. W. Reversible Switching of Ultrastrong Light-Molecule Coupling. Phys. Rev. Lett. 2011, 106, 196405.

(14) Lin, L.; Wang, M.; Wei, X.; Peng, X.; Xie, C.; Zheng, Y. Photoswitchable Rabi Splitting in Hybrid Plasmon-Waveguide Modes. Nano Letters 2016, 16, 7655-7663.

(15) Baudrion, A.-L.; Perron, A.; Veltri, A.; Bouhelier, A.; Adam, P.-M.; Bachelot, R. Reversible Strong Coupling in Silver Nanoparticle Arrays Using Photochromic Molecules. Nano Letters 2013, 13, 282-286.

(16) Slavov, C.; Boumrifak, C.; Hammer, C. A.; Trojanowski, P.; Chen, X.; Lees, W. J.; Wachtveitl, J.; Braun, M. The Ultrafast Reactions in the Photochromic Cycle of WaterSoluble Fulgimide Photoswitches. Phys. Chem. Chem. Phys. 2016, 18, 10289-10296.

(17) Kuwata, H.; Tamaru, H.; Esumi, K.; Miyano, K. Resonant Light Scattering from Metal 
Nanoparticles: Practical Analysis Beyond Rayleigh Approximation. Applied Physics Letters 2003, 83, 4625-4627.

(18) Pala, R. A.; Shimizu, K. T.; Melosh, N. A.; Brongersma, M. L. A Nonvolatile Plasmonic Switch Employing Photochromic Molecules. Nano Letters 2008, 8, 1506-1510.

(19) Nishi, H.; Asahi, T.; Kobatake, S. Light-Controllable Surface Plasmon Resonance Absorption of Gold Nanoparticles Covered with Photochromic Diarylethene Polymers. The Journal of Physical Chemistry C 2009, 113, 17359-17366.

(20) Ming, T.; Zhao, L.; Xiao, M.; Wang, J. Resonance-Coupling-Based Plasmonic Switches. Small 2010, 6, 2514-2519.

(21) Zheng, Y. B.; Kiraly, B.; Cheunkar, S.; Huang, T. J.; Weiss, P. S. Incident-AngleModulated Molecular Plasmonic Switches: A Case of Weak Exciton-Plasmon Coupling. Nano Letters 2011, 11, 2061-2065.

(22) Sridharan, D.; Bose, R.; Kim, H.; Solomon, G. S.; Waks, E. A Reversibly Tunable Photonic Crystal Nanocavity Laser Using Photochromic Thin Film. Opt. Express 2011, $19,5551-5558$.

(23) Furumi, S.; Fudouzi, H.; Sawada, T. Dynamic Photoswitching of Micropatterned Lasing in Colloidal Crystals by the Photochromic Reaction. J. Mater. Chem. 2012, 22, 2151921528.

(24) Kobayashi, T. J-Aggregates; World Scientific Publishing: Hackensack, NJ, USA, 1996.

(25) Vasa, P.; Pomraenke, R.; Cirmi, G.; De Re, E.; Wang, W.; Schwieger, S.; Leipold, D.; Runge, E.; Cerullo, G.; Lienau, C. Ultrafast Manipulation of Strong Coupling in MetalMolecular Aggregate Hybrid Nanostructures. ACS Nano 2010, 4, 7559-7565.

(26) Schlather, A. E.; Large, N.; Urban, A. S.; Nordlander, P.; Halas, N. J. Near-Field 
Mediated Plexcitonic Coupling and Giant Rabi Splitting in Individual Metallic Dimers. Nano Letters 2013, 13, 3281-3286.

(27) Zhang, K.; Chen, T.-Y.; Shi, W.-B.; Li, C.-Y.; Fan, R.-H.; Wang, Q.-J.; Peng, R.W.; Wang, M. Polarization-Dependent Strong Coupling Between Surface Plasmon Polaritons and Excitons in an Organic-Dye-Doped Nanostructure. Opt. Lett. 2017, 42, $2834-2837$.

(28) Baieva, S.; Hakamaa, O.; Groenhof, G.; Heikkilä, T. T.; Toppari, J. J. Dynamics of Strongly Coupled Modes Between Surface Plasmon Polaritons and Photoactive Molecules: The Effect of the Stokes Shift. ACS Photonics 2017, 4, 28-37.

(29) Vasa, P.; Wang, W.; Pomraenke, R.; Lammers, M.; Maiuri, M.; Manzoni, C.; Cerullo, G.; Lienau, C. Real-time observation of ultrafast Rabi oscillations between excitons and plasmons in metal nanostructures with J-aggregates. Nature Photonics 2013, \%, 128-132.

(30) Qin, C.; Gao, Y.; Qiao, Z.; Xiao, L.; Jia, S. Atomic-Layered MoS2 as a Tunable Optical Platform. Advanced Optical Materials 2016, 4, 1429-1456.

(31) Butun, S.; Tongay, S.; Aydin, K. Enhanced Light Emission from Large-Area Monolayer $\mathrm{MoS}_{2}$ Using Plasmonic Nanodisc Arrays. Nano Lett 2015, 15, 2700-2704.

(32) Liu, W.; Lee, B.; Naylor, C. H.; Ee, H.-S.; Park, J.; Johnson, A. T. C.; Agarwal, R. Strong Exciton-Plasmon Coupling in $\mathrm{MoS}_{2}$ Coupled with Plasmonic Lattice. Nano Letters 2016, 16, 1262-1269.

(33) Zu, S.; Li, B.; Gong, Y.; Li, Z.; Ajayan, P. M.; Fang, Z. Active Control of PlasmonExciton Coupling in MoS2-Ag Hybrid Nanostructures. Advanced Optical Materials 2016, 4, 1463-1469. 
(34) Li, Z.; Xiao, Y.; Gong, Y.; Wang, Z.; Kang, Y.; Zu, S.; Ajayan, P. M.; Nordlander, P.; Fang, Z. Active Light Control of the MoS2 Monolayer Exciton Binding Energy. ACS Nano 2015, 9, 10158-10164.

(35) Sukharev, M. Control of Optical Properties of Hybrid Materials with Chirped Femtosecond Laser Pulses Under Strong Coupling Conditions. The Journal of Chemical Physics 2014, 141, 084712.

(36) Demetriadou, A.; Hamm, J. M.; Luo, Y.; Pendry, J. B.; Baumberg, J. J.; Hess, O. Spatiotemporal Dynamics and Control of Strong Coupling in Plasmonic Nanocavities. ACS Photonics 2017, (Just Accepted).

(37) Zhao, W.; Wang, S.; Liu, B.; Verzhbitskiy, I.; Li, S.; Giustiniano, F.; Kozawa, D.; Loh, K. P.; Matsuda, K.; Okamoto, K.; Oulton, R. F.; Eda, G. Exciton-Plasmon Coupling and Electromagnetically Induced Transparency in Monolayer Semiconductors Hybridized with Ag Nanoparticles. Advanced Materials 2016, 28, 2709-2715.

(38) Lee, B.; Liu, W.; Naylor, C. H.; Park, J.; Malek, S. C.; Berger, J. S.; Johnson, A. T. C.; Agarwal, R. Electrical Tuning of Exciton-Plasmon Polariton Coupling in Monolayer MoS2 Integrated with Plasmonic Nanoantenna Lattice. Nano Letters 2017, 17, 45414547.

(39) Li, B.; Zu, S.; Zhou, J.; Jiang, Q.; Du, B.; Shan, H.; Luo, Y.; Liu, Z.; Zhu, X.; Fang, Z. Single-Nanoparticle Plasmonic Electro-optic Modulator Based on MoS2 Monolayers. ACS Nano 2017, (Just Accepted).

(40) Kang, Y.; Najmaei, S.; Liu, Z.; Bao, Y.; Wang, Y.; Zhu, X.; Halas, N. J.; Nordlander, P.; Ajayan, P. M.; Lou, J.; Fang, Z. Plasmonic Hot Electron Induced Structural Phase Transition in a MoS2 Monolayer. Advanced Materials 2014, 26, 6467-6471.

(41) Wen, J.; Wang, H.; Wang, W.; Deng, Z.; Zhuang, C.; Zhang, Y.; Liu, F.; She, J.; Chen, J.; Chen, H.; Deng, S.; Xu, N. Room-Temperature Strong Light-Matter Interac- 
tion with Active Control in Single Plasmonic Nanorod Coupled with Two-Dimensional Atomic Crystals. Nano Letters 2017, 17, 4689-4697.

(42) Khatua, S.; Chang, W.-S.; Swanglap, P.; Olson, J.; Link, S. Active Modulation of Nanorod Plasmons. Nano Letters 2011, 11, 3797-3802.

(43) Chang, W.-S.; Lassiter, J. B.; Swanglap, P.; Sobhani, H.; Khatua, S.; Nordlander, P.; Halas, N. J.; Link, S. A Plasmonic Fano Switch. Nano Letters 2012, 12, 4977-4982.

(44) Kossyrev, P. A.; Yin, A.; Cloutier, S. G.; Cardimona, D. A.; Huang, D.; Alsing, P. M.; $\mathrm{Xu}$, J. M. Electric Field Tuning of Plasmonic Response of Nanodot Array in Liquid Crystal Matrix. Nano Letters 2005, 5, 1978-1981.

(45) Dickson W., E. P. P. R. Z. A., Wurtz G.A. Electronically Controlled Surface Plasmon Dispersion and Optical Transmission Through Metallic Hole Arrays Using Liquid Crystal. Nano Letters 2007, 6, 281-286.

(46) Evans, P. R.; Wurtz, G. A.; Hendren, W. R.; Atkinson, R.; Dickson, W.; Zayats, A. V.; Pollard, R. J. Electrically Switchable Nonreciprocal Transmission of Plasmonic Nanorods with Liquid Crystal. Applied Physics Letters 2007, 91, 043101.

(47) Chung-Tse, C.; Chun-Chih, L.; Chun-Hsiung, W.; Chih-Wei, C.; Yang-Fang, C. Tunable Coupling Between Exciton and Surface Plasmon in Liquid Crystal Devices Consisting of Au Nanoparticles and CdSe Quantum Dots. Applied Physics Letters 2011, 98, 261918.

(48) Si, G.; Zhao, Y.; Leong, E. S. P.; Liu, Y. J. Liquid-Crystal-Enabled Active Plasmonics: A Review. Materials 2014, 7, 1296-1317.

(49) Minamimoto, H.; Kato, F.; Nagasawa, F.; Takase, M.; Murakoshi, K. Electrochemical Control of Strong Coupling States Between Localized Surface Plasmons and Molecule Excitons for Raman Enhancement. Faraday Discussions 2017, Advance Article. 
(50) Miyata, M.; Kaijima, A.; Nagasaki, Y.; Takahara, J. Electromechanically Tunable Plasmonic Nanowires Operating in Visible Wavelengths. ACS Photonics 2016, 3, 22682274 .

(51) Bedogni, E.; Kaneko, S.; Fujii, S.; Kiguchi, M. Mechanical Control of the Plasmon Coupling with Au Nanoparticle Arrays Fixed on the Elastomeric Film via Chemical Bond. Japanese Journal of Applied Physics 2017, 56, 035201.

(52) Cetin, A. E.; Mertiri, A.; Huang, M.; Erramilli, S.; Altug, H. Thermal Tuning of Surface Plasmon Polaritons Using Liquid Crystals. Advanced Optical Materials 2013, $1,915-920$.

(53) Joshi G.K., J. M. S. R., Smith K.A. Temperature Controlled Reversible Localized Surface Plasmon Resonance Response of Polymer-Functionalized Gold Nanoprisms in the Solid State. J. Phys. Chem. C 2013, 117, 26228-37.

(54) Jostmeier, T.; Mangold, M.; Zimmer, J.; Karl, H.; Krenner, H. J.; Ruppert, C.; Betz, M. Thermochromic Modulation of Surface Plasmon Polaritons in Vanadium Dioxide Nanocomposites. Opt. Express 2016, 24, 17321-17331.

(55) Beebe, M.; Wang, L.; Madaras, S. E.; Klopf, J. M.; Li, Z.; Brantley, D.; Heimburger, M.; Wincheski, R. A.; Kittiwatanakul, S.; Lu, J.; Wolf, S. A.; Lukaszew, R. A. Surface Plasmon Resonance Modulation in Nanopatterned Au Gratings by the Insulator-Metal Transition in Vanadium Dioxide Films. Opt. Express 2015, 23, 13222-13229.

(56) Abate, Y.; Marvel, R. E.; Ziegler, J. I.; Gamage, S.; Javani, M. H.; Stockman, M. I.; Haglund, R. F. Control of Plasmonic Nanoantennas by Reversible Metal-Insulator Transition. Nature Scientific Reports 5.

(57) Lopez, R.; Haynes, T. E.; Boatner, L. A.; Feldman, L. C.; Haglund, R. F. TemperatureControlled Surface Plasmon Resonance in VO2 Nanorods. Opt. Lett. 2002, 27, 13271329. 
(58) Cliento, F.; Giannetti, C.; Ferrini, G.; Dal Conte, S.; Sala, T. Ultrafast Insulator-toMetal Phase Transition as a Switch to Measure the Spectrogram of a Supercontinuum Light Pulse. Appl. Phys. Lett. 2010, 96, 431-434.

(59) Wegkamp, D.; Herzog, M.; Xian, L.; Gatti, M.; Cudazzo, P.; McGahan, C. L.; Marvel, R. E.; Haglund, R. F.; Rubio, A.; Wolf, M.; Stähler, J. Instantaneous Band Gap Collapse in Photoexcited Monoclinic $\mathrm{VO}_{2}$ due to Photocarrier Doping. Phys. Rev. Lett. 2014, 113, 216401.

(60) Cao, J.; Ertekin, E.; Srinivasan, V.; Fan, W.; Huang, S.; Zheng, H.; Yim, J. W. L.; Khanal, D. R.; Ogletree, D. F.; Grossman, J. C.; Wu, J. Strain Engineering and One-Dimensional Organization of Metal-Insulator Domains in Single-Crystal Vanadium Dioxide Beams. Nature Nanotechnology 2009, 4, 732-737.

(61) Park, J. H.; Coy, J. M.; Kasirga, T. S.; Huang, C.; Fei, Z.; Hunter, S.; Cobden, D. H. Measurement of a Solid-State Triple Point at the Metal-Insulator Transition in VO2. Nature 2013, 500, 431-434.

(62) Berrier, A.; Cools, R.; Arnold, C.; Offermans, P.; Crego-Calama, M.; Brongersma, S. H.; Gomez-Rivas, J. Active Control of the Strong Coupling Regime Between Porphyrin Excitons and Surface Plasmon Polaritons. ACS Nano 2011, 5, 6226-6232.

(63) Zheng, D.; Zhang, S.; Deng, Q.; Kang, M.; Nordlander, P.; Xu, H. Manipulating Coherent Plasmon-Exciton Interaction in a Single Silver Nanorod on Monolayer WSe2. Nano Letters 2017, 17, 3809-3814.

(64) Wang, Z.; Dong, Z.; Gu, Y.; Chang, Y.-H.; Zhang, L.; Li, L.-J.; Zhao, W.; Eda, G.; Zhang, W.; Grinblat, G.; Maier, S. A.; Yang, J. K. W.; Qiu, C.-W.; Wee, A. T. S. Giant Photoluminescence Enhancement in Tungsten-Diselenide-Gold Plasmonic Hybrid Structures. Nature Communications 2016, 7. 
(65) Ding, T.; Sigle, D.; Zhang, L.; Mertens, J.; de Nijs, B.; Baumberg, J. Controllable Tuning Plasmonic Coupling with Nanoscale Oxidation. ACS Nano 2015, 9, 6110-6118. 
For Table of Contents Only
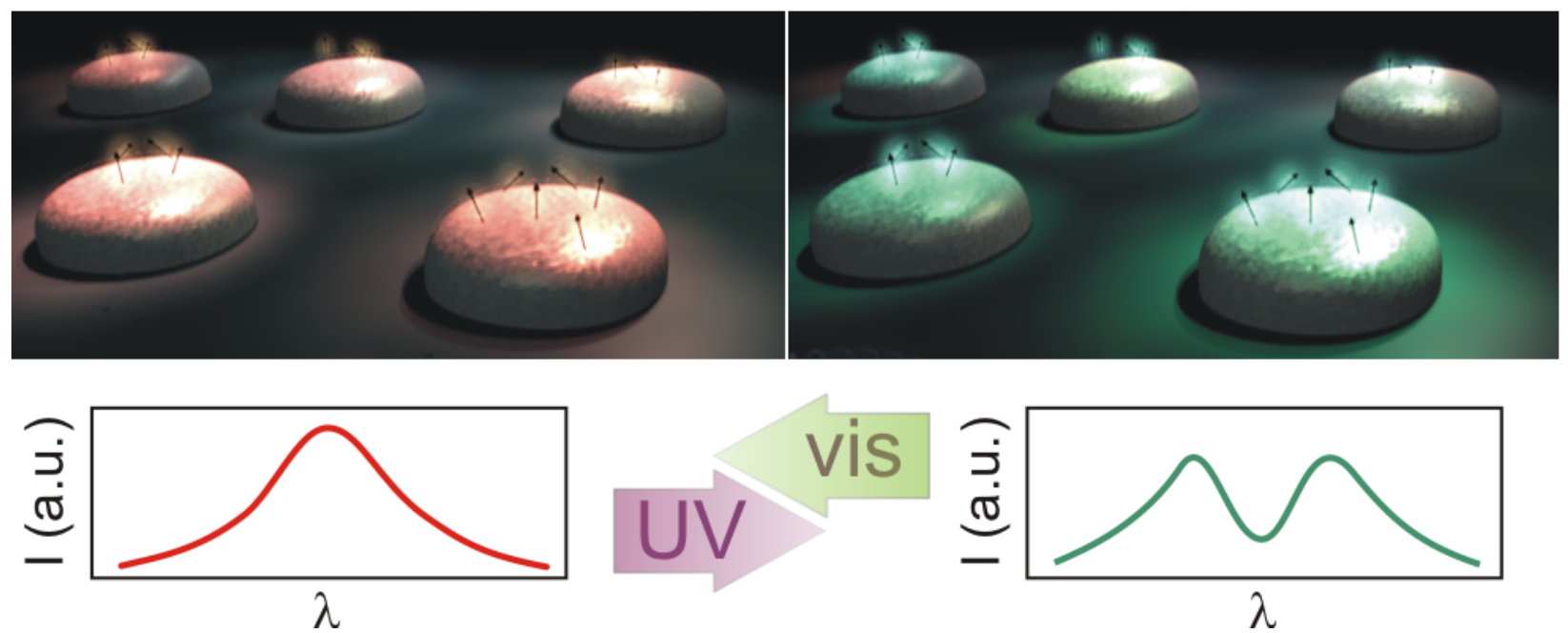